\title{
Cervical Dystonia in Pregnancy in a Woman with Three Kidneys, Uterine and Ocular Anomalies
}

\author{
Madu AE
}

Department of Obstetrics and Gynaecology, Staffordshire General Hospital, Mid Staffordshire Hospitals NHS Trust, Stafford, United Kingdom.

Pregnancy complicated by rare or uncommon neurological disorders of movement can present to the obstetrician/staff and thus present a challenge to the routine obstetric care staff. A case of a 30 - year- primigravida with cervical dystonia, ocular, genital and renal tract anomaly is presented with a literature review.

Keywords: cervical dystonia, pregnancy, renal tract anomaly.

\section{INTRODUCTION}

Cervical dystonia is a distressing chronic neurological movement disorder of neurological origin. It causes the neck to involuntarily turn to the left, right, upwards, and/or downwards. Agonist and antagonist muscles contract at the same time in dystonic movement. ${ }^{1}$ It is mainly idiopathic but can be secondary to an underlying disorder. Most sufferers have the first episode in midlife. Severe cervical dystonia in pregnancy and its management can be distressing to the patient and can influence pregnancy outcomes. Dystonia can be difficult to diagnose, as it can simulate other neurological diseases. Genital tract anomalies are associated with renal tract anomaly in $30 \%$ of cases and can also influence pregnancy outcomes. This is a case of dystonia in pregnancy in a woman with associated ocular, renal and genital tract anomalies and it is speculated whether these problems constitute a new syndrome yet to be characterized and recognized.

\section{CASE}

A 30-year-old primigravida was booked for antenatal care in our unit at 10 weeks gestation with a normal dating scan. Her blood group was A-positive with no atypical antibodies. Routine test for renal function was normal and so was infections screening. Her body mass index was 20 . She had a history of

\section{CORRESPONDENCE}

Dr Anthony Emeka Madu

Department of Obstetrics and Gynaecology,

Staffordshire General Hospital, Mid Staffordshire Hospitals NHS Trust, Stafford, United Kingdom.

Email: emymadu@yahoo.co.uk

Phone: 00447931626315 recurrent urinary tract infection (UTI) as a child and investigations revealed 3 kidneys; a duplicate kidney on her left side. She had cystoscopy many times and urethral dilatation twice. The re-occurrence of UTI reduced markedly as she grew up. She is a smoker. She suffered depression, was on antidepressant prior to conception and stopped medication on discovering she was pregnant. She drank alcohol before conception but reduced to half a unit on discovering she was pregnant. Previous ocular examination was normal apart from the right eye being bigger than the left. Her mother had a history of hypothyroidism.

At 17 weeks gestation, she gradually developed stiffness and involuntary recurrent spasms of the neck, stiffness and pain of the left arm and shoulder. The muscles involved sternocleidomastoids with upward and downward movements of the neck. The dystonia got worse and the associated pain did not improve while taking simple analgesics. Her pain got gradually worse over the next two weeks and she could no longer work. She was diagnosed with arthritis in her neck and was told it was probably due to a car accident she had at the age of 18 that was associated with whiplash injury. Fetal anomaly scan was normal at 20 weeks gestation.

She was then referred to neurologist who made the diagnosis, recommended Magnetic Resonance Imaging (MRI) scan and prescribed standard doses

The papers in this journal are published under the terms of the Creative Commons Attribution License. Users are allowed to read, download, copy, distribute, print, search, or link to the full texts of the articles in this journal without asking prior permission from the publisher or the author. 
of codeine phosphate and paracetamol. MRI of the spine reported no pathology. At this stage, she was in distress and could barely walk. She was sleeping in a chair as she was unable to get up the stairs to her bed, and also unable to lie down. She felt her spine had twisted and her left shoulder appeared to have risen up towards her neck. She was also having painful spasms in her neck and shoulder if she moved had the affected side touched. The pain was also present in left arm, particularly around her wrist. She could only take paracetamol, was not getting sleep and was not gaining any weight. Her brain MRI scan was normal. A 24-hour urine sample showed a raised level of copper but without any derangement of liver or renal function. She later developed some sensory tricks (geste antagoniste; touching her chin) that she thought could relieve her symptoms, but all to no avail.

At $35+{ }^{4}$ weeks gestation, she presented to labour ward with history of spontaneous membrane rupture which was confirmed on vaginal speculum examination. She was admitted for observation. The next day $35+^{5}$ gestation she went into established labour and delivered a baby boy with good Apgar score weighing 1990 gm. Placenta and cord vessels were normal.

A large painless poorly defined lump on the left side of the neck was noted at about three days following delivery. MRI scan was arranged for 6 weeks postdelivery following a surgical input. There were no maternal and infant concerns at 6-weeks follow up and urinary copper level was only a trace. MRI of the neck following delivery was normal. Pelvic ultrasound 6 weeks following delivery showed bicornuate uterus.

\section{COMMENT}

Dystonia is a syndrome of abnormal sustained muscle contraction that produces repetitive involuntary twisting movements and abnormal posturing of neck, trunk, face and extremities. It may be focal segmental or generalised and there are many subdivisions. ${ }^{2}$ Causes can be hormonal abnormality, brain injury (damage to basal ganglia), medication or genetic. Hereditary dystonia is apparent from 5 to 15 years of age. Dystonic movements may be precipitated or increased by stress as in this patient, and helped with relaxation.

Prevalence of dystonia affecting many parts of the body is $0.2-0.6 \%$ per 100,000 and that affecting one part of the body is 30 per $100,000 .^{2}$ The exact prevalence of the disorder is unknown as several family and population studies show that up to $25 \%$ of cervical dystonia patients have relatives that are undiagnosed. ${ }^{3,4}$ Females have a higher prevalence compared to males to a ratio of 1.5 times. The prevalence of dystonia also increases with age and most patients show symptoms from ages $50-69$. The average age of onset is 41 . $^{1}$

Dystonia is classified with respect to age of onset, location and aetiology, but the latter two appear to be more relevant. Dystonia can be primary (inherited) or secondary (adult forms). The latter usually present in later life. Primary forms are usually genetic; autosomal dominance with reduced penetrance demonstrated in DYT1 gene. ${ }^{5}$ Our patient gave no such family history. Studies have shown the DYT7 locus on chromosome $18 \mathrm{p}$ in a German family and the DYT13 locus on chromosome 1 p36 in an Italian family is associated with dystonia. Inheritance for both loci is autosomal dominant with reduced penetrance. These loci have been found, but is still unclear the extent of influence the loci has on spasmodic torticollis. ${ }^{6}$

Secondary cervical dystonia is usually due to exogenous insults, neurological anomalies other than those of cervical dystonia, anomalies of brain especially in the basal ganglia. We excluded perinatal brain injury, kernicterus, cerebrovascular disease, drug induced, toxins, metabolic, central pointine myelinosis, peripheral and central trauma, infectious and post infectious encephalopathies.

Cervical dystonia mimics Jacksonian epilepsy, benign essential tremor, Wilson's disease, Sydenham's chorea, Huntington's disease and wry neck whiplash injury. It can cause diagnostic confusion, as the diagnosis of dystonia is essentially clinical. Codydramol may have helped the patient but it also could have been coincidental and some cases are known to regress without medical intervention. Tardive dystonia is usually secondary to medication like anticonvulsants, antipsychotics, metoclopramide, dopamine agonist, calcium channel blocker, etc. It is the commonest form of secondary adult dystonia and is found among European and Ashkenazi Jews. Our patient was not on these medications. Dystonia can be of psychogenic origin usually acute in onset as in this patient especially secondary to stressful events in the patient's life.

Torticollis of dystonia can be rotational (rotational collis/chin-to-shoulder version); laterocollis (the tilting of the head from side to side/ear-to-shoulder version), anterocollis (head tilts forward/chin-to-chest version); the latter being the most difficult version to treat and presented in my case, and retrocollis (the extension of the neck, head tilts back). A combination of these head positions is common; many patients experience turning and tilting actions of the head. Our patient may have benefited from benzodiazepines but this was not part of her treatment. There has 
been a reported case of 31 -year- Chinese woman with cervical dystonia gravidarum treated with clonazepam. ${ }^{8}$ The latter was stopped in the second trimester and the dystonia did not recur after tapering off the dose and then stopping the clonazepam. The first case of torsion dystonia was appeared to have been in a 35 -year- primigravida who presented for elective caesarean section and thus demonstrated the challenges faced by medical staff treating these patients during delivery.

Our patient presented with urinary excretion of copper mimicking Wilson's disease. However, abdominal distension, splenomegaly, speech impairment, jaundice and hepatic impairment, delirium, dementia that are common in Wilson's disease were not present in our patient. Excretion of copper disappeared to a trace 6 weeks after delivery.

Treatment of dystonia should be individualised and each woman's case is unique as demonstrated in this patient. Surgical procedures like ramisectomy or rhizotomy are denervation procedures that can be selective. The latter are rarely performed for dystonia. Complimentary therapies like chiropractor adjustments may be associated with harm due to the nature of dystonia. Gentle massage may only give temporary relief. Generally, braces or orthopaedic devices do not help and may make the situation worse because of spasm or twist of the affected part against or into the brace. Acupuncture may help relieve pain and benefit may be cumulative from anecdotal reports. ${ }^{9}$ It can also be used with traditional drugs without adverse outcomes. Hypnosis does not treat dystonia although relaxation techniques help improve symptoms and quality of life.

No strong relationship exists between dystonia and diet. However, nicotine, caffeine or related stimulants may worsen symptoms in some patients. Alcohol can have dual effect; being of some relief in some situation and worsen in others. Vitamins and nutritional supplements have not shown to effect significant improvement. Inducing calmness, sleepiness or other forms of relaxation can mildly relieve symptoms.

The incidence of renal tract involvement in uterine anomaly vary from 5 to $100 \%$ or average of $30 \% .^{10}$ It was not surprising to find uterine anomaly in this patient. I had speculated that all the problems this patient had in her medical and obstetrical history could be or related to a rare syndrome yet to be known to the medical community. However, an extensive literature search did not yield any article on any relationship of genitourinary anomalies to ocular anomalies or any association of the former or the latter with dystonia in pregnancy. There is no reported syndromic entity encompassing cervical dystonia, ocular, genital and urinary abnormalities. Thus this unique patient presented with unusual challenges and apparently at the time clearly redefined the limits of our thinking of obstetric and medical care.

\section{REFERENCES}

1. Geyer HL, Bressman SB. The diagnosis of dystonia. Lancet Neurol. 2006;5(9):780-90.

2. Adler CH. Strategies for controlling dystonia: overview of therapies that may alleviate symptoms. Postgrad Med. 2000;108(5):15-60.

3. Waddy HM, Fletcher NA, Harding AE, Marsden CD. A genetic study of idiopathic focal dystonias. Ann Neurol. 1991;29(3):320-4

4. Duffey PO, Butler AG, Hawthorne MR, Barnes MP. The epidemiology of the primary dystonias in the north of England. Adv Neurol. 1998;78:121-5.

5. Olufolabi AJ, Wee MYK. Caesarean section in a patient with torsion dystonia. Br J Anaesth. 2006;96(5):611-3.

6. de Carvalho Aguiar PM, Ozelius LJ. Classification and genetics of dystonia. Lancet Neurol. 2002;1(5):316-25.

7. Brashear A. Treatment of cervical dystonia with botulinum toxin. Operative Techniques in Otolaryngology-Head and Neck Surgery. 2004;15(2):122-7.

8. Lim EC, Seet RC, Wilder-Smith EPV, Ong BK. Dystonia gravidarum: a new entity? Mov Disord. 2005;21(1):60-70.

9. Dystonia Medical Research Foundation. [cited 2012 October 12]. Available from http://www.dystonia-foundation.org/ pages/frequently asked question/159.php

10. Tan SL, Tulandi T. Reproductive endocrinology and infertility: current trends and development. New York: Marcel Dekker; 2003.p.225. 